\title{
VARIABLES THAT AFFECT RESULTS OF PFA-100 IN A GROUP OF HEALTHY BLOOD DONORS IN THE SLOVAK POPULATION
}

\author{
KORINKOVA L ${ }^{1}$, STASKo J ${ }^{1}$, KUBISZ P1 , GRENDAR M. ${ }^{2}$ \\ ${ }^{1}$ National Centre of Hemostasis and Thrombosis, Clinic of Hematology and Transfusiology, Comenius University in \\ Bratislava, Jessenius Faculty of Medicine in Martin and University Hospital in Martin, Slovakia. \\ ${ }^{2}$ Biomedical Center Martin, Department of Bioinformatics, Comenius University in Bratislava, Jessenius Faculty of \\ Medicine, Martin, Slovakia
}

\begin{abstract}
Background: The platelet function analyzer (PFA-100) is a system analyzing platelet function determined for detection of the functional inherited and acquired platelet disorders, screening of von Willebrand disease (vWD) and recently also considered as useful for monitoring of antiplatelet treatment. The PFA-100 test uses a high shear flow system to simulate in vitro the conditions to which platelets are subjected at the site of a damaged blood vessel wall.

Aim of study: We decided to establish the reference intervals of PFA closure time (CT) in the Slovak population of healthy blood donors.

Patients and methods: Fifty age and gender matched healthy blood donors were enrolled in the study. We investigated the relationships between PFA-100 CT, gender and ABO blood groups.

Results: The reference intervals for CT measured on CEPI (collagen/epinephrine) and CADP (collagen/adenosine diphosphate) cartridge in 3.2\% citrated blood were 86 - 199 sec. and $42-119$ sec., respectively. Blood group O was associated with significantly longer CEPI CT $(\mathrm{p}<0.05)$ compared to non - O groups. The prolongation of CADP CT in blood donors with blood group $\mathrm{O}$ was without significance. The influence of gender as another variable analyzed with CT has not been evaluated as statistically significant.

Conclusion: PFA-100 CT should be interpreted carefully with consideration of both the patient's clinical presentation and laboratory variables such as $\mathrm{ABO}$ blood group.
\end{abstract}

Keywords: platelet function analyzer (PFA-100), closure time (CT), collagen/epinephrine (CEPI), collagen/ADP (CADP), blood group, gender.

\section{INTRODUCTION}

Platelets play a pivotal role in both hemostasis and thrombosis. Primary hemostasis involves platelet adhesion to subendothelial tissue of the injured vessel wall. Under high shear stress, von Willebrand factor (vWF) acts as the mediator for both platelet adhesion and aggregation. Adhesion of platelets to collagen-containing lesions in the vessel wall results in activation of the platelets, release of various platelet activating mediators, and ultimately leads to augmented platelet aggregation and formation of a primary hemostatic plug [1,2] ( Fig. 1).

Accurate measurements of platelet function are important, both for screening platelet dysfunction and for monitoring response to anti-platelet therapy. Since its introduction, platelet aggregometry has become the standard method for testing platelet function. Conventional platelet aggregometry has many limitations including being time and labor-intensive and operator-dependent, and requiring the preparation of platelet-rich plasma. Furthermore, the testing and interpretation require considerable experience [2].

Recently, a platelet function analyzer (PFA-100, Dade Behring) was introduced that simulates in vivo hemostatic plug formation. This simple, rapid, in vitro method aids in the detection of platelet dysfunction [3]. Briefly, citrated whole blood is aspirated at a high shear rate from a sample reservoir through a $150 \mu \mathrm{m}$ aperture in a membrane coated with collagen and epinephrine

\footnotetext{
Address for correspondence:

Lubica Korinkova, MSc, Clinic of Hematology and Transfusiology, Comenius University in Bratislava, Jessenius Faculty of Medicine in Martin, University Hospital, Kollarova 2, 03601 Martin, Slovakia e-mail: korinkova@jfmed.uniba.sk
} 
Primary Hemostasis

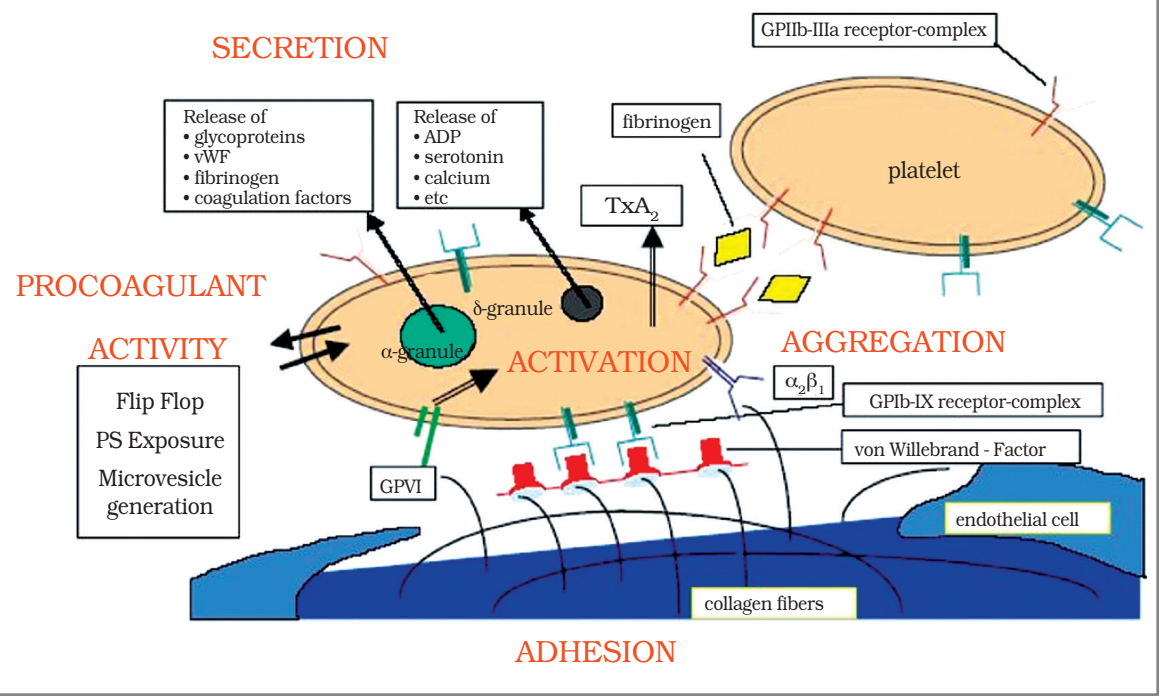

Fig. 1 Pivotal players and actions of primary hemostasis [1]

(CEPI), or collagen and adenosine diphosphate (ADP) (CADP). Mediated by vWF, platelets adhere to the collagen on the membrane, which then activates the platelets, causing them to aggregate in and around the aperture, a process that eventually occludes the aperture. Results of PFA100 testing are reported as closure times (CT), or the time in seconds (sec.) required for the aperture to occlude. CT provides a measure of overall platelet-associated primary hemostasis, with shorter closure time indicative of higher platelet function.

Several pre-analytical variables affect PFA-100 test results [4-13]. CT depend on the plasma vWF level, citrate concentration, ABO blood group, and time of blood collection. In addition a low platelet count, or low hematocrit may lead to prolongation of CT [7-11]. To determine PFA-100 reference intervals in the Slovak population, we evaluated CEPI and CADP closure times in 50 well-characterized healthy blood donors. We also studied the relationships between PFA-100 $\mathrm{CT}$, gender and $\mathrm{ABO}$ blood group in these subjects.

\section{MATERIAL AND METHODS}

Fifty apparently healthy Slovak adults (> 18 year old) were enrolled in this study. All files were investigated in healthy blood donors of the National Transfusion Service in Martin. In the study group, there were 38 men and 12 women. The average age of the group was 38.8 years, the average age of men 38.2 years and women 39.9 years. In the file there were represented basically all blood groups. The most numerous blood group was $0(46 \%)$. There were $32 \%$ of blood donors with blood group $\mathrm{A}$ in the file, $20 \%$ with blood group $\mathrm{B}$ and $2 \%$ with blood group $\mathrm{AB}$, respectively (Fig.2). Estimated data of the percentage of blood groups in Slovakia according to Slovak National Transfusion Service [unpublished results], is as follows: A - 48\%, 0-39\%, B - 9\%, $\mathrm{AB}-4 \%$ (Fig.3). Each donation of blood was examined by complete blood counts (WBC, RBC, PLT, HTC) using the blood cell analyzer Sysmex KX-2 1 (SIEMENS). All subjects were considered as healthy individuals with no previous history or laboratory results indicating platelet dysfunctions. Individuals with history of bleeding disorders, liver disease and renal insufficiency, or if they've been taking aspirin, aspirin-containing compounds, ibuprofen, antibiotics, antihistamines, non-steroidal anti-inflammatory drugs, oral anticoagulants, or any other drugs that affect platelet function were excluded. Other exclusion criteria included platelet count 
$<150.000 / \mu 1$, or hematocrit $<35 \%$. Study was approved by Ethic Committee of JFM CU and each subject had given written informed consent. Blood was drawn into a tube containing $3.2 \%$ $(0.109 \mathrm{M})$ buffered sodium citrate solution. The blood sample was analyzed within 20 min in the PFA-100 for CT with both the EPI and ADP cartridges.

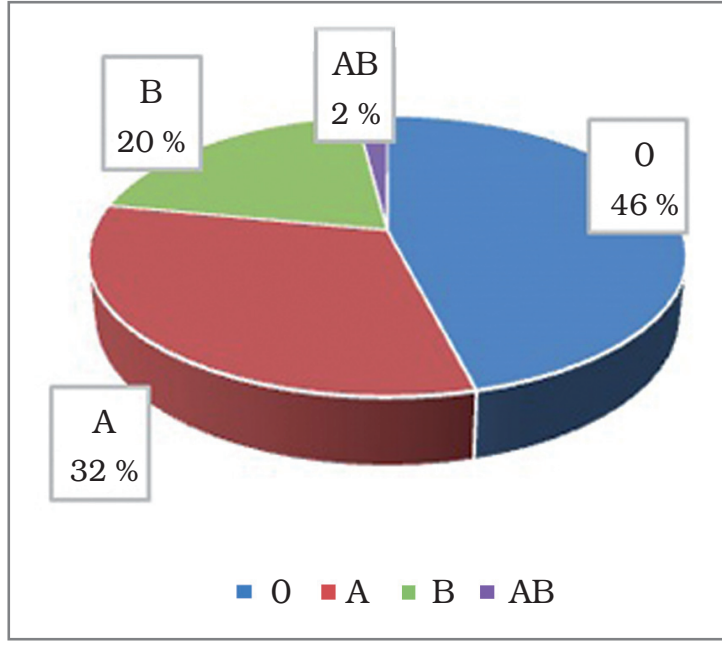

Fig.2 ABO blood group presentation in the investigated population.

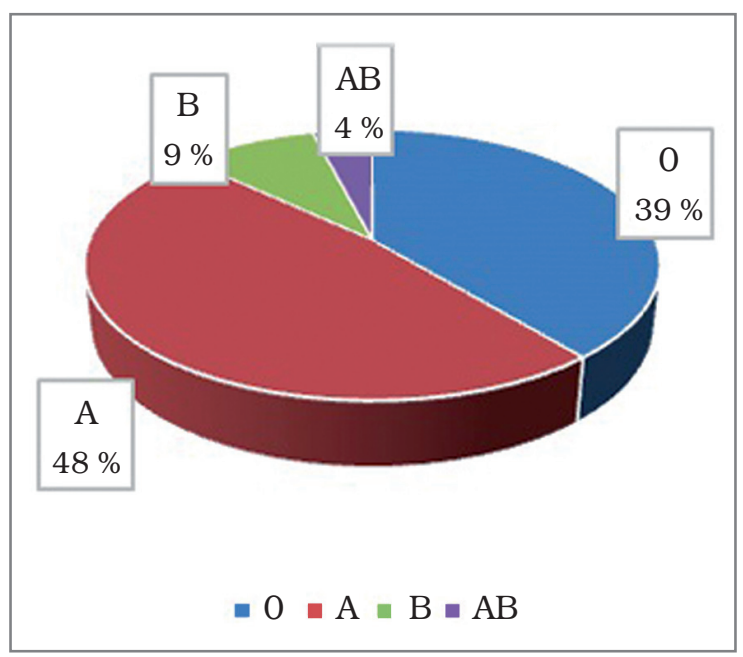

Fig.3 The estimated percentage of $\mathrm{ABO}$ blood group in Slovakia [unpublished data]

Statistical analysis: Values are presented either as mean \pm standard deviation (SD) or as median, range and interquartile range (IQR). Correlation of CT between both the patients with blood group $\mathrm{O}$ and blood group non $\mathrm{O}$ as well as correlation of CT between group of men and women were evaluated using paired Student's t-test and Wilcoxon Signed-Rank test.

Specimen collection: Blood samples were taken from each subject's antecubital vein using the BD Vacutainer system (Becton-Dickinson). The initial small amount of whole blood was collected into EDTA collection tubes for complete blood count (CBC) determination. Samples for PFA-100 testing were drawn into $0.109 \mathrm{M}(3.2 \% \mathrm{w} / \mathrm{v})$ sodium citrate collection tubes and stored at room temperature prior to testing. Hemolyzed or clotted samples were not used.

PFA-100: The PFA-100® system (Dade Behring) is a desktop analyzer, which can be used to quickly obtain valuable information about the status of primary hemostasis. The PFA-100® instrument has been recently updated to PFA-200 [14]. It is composed of a microprocessor-controlled device and single-use test cartridges. Three cartridges are currently available; the CADP cartridge contains collagen and ADP, the CEPI cartridge contains collagen and epinephrine, and the INNO-


P2Y cartridge was developed in view of the relative insensitivity of previous cartridges to P2Y $\mathrm{Y}_{12}$ receptor inhibitors, and appears promising in evaluating congenital P2Y12 defects as well as in monitoring P2Y12 receptor inhibitors [15]. The CEPI and CADP test cartridges consist of a sample reservoir, a capillary and a membrane coated with platelet agonists $(2 \mathrm{mg}$ equine type I collagen and either 10 mg epinephrine bitartrate - CEPI cartridge or 50 mg ADP - CADP cartridge) [16].

PFA-100 device comprises a carousel for the positioning of two measuring cartridges, LCD display, keyboard, printer and a box for a starting solution. Cartridges are inserted into the carousel, in which the measuring cartridge is inserted. Once the examined sample is pipetted into the cartridge, press the RUN button on the LCD. The carousel starts rotating clockwise and deliver measuring cartridge into the device. The device leads to the identification of measuring cartridges, addition of starter solution, sample incubation and test completion. (Fig.4) [16]. 


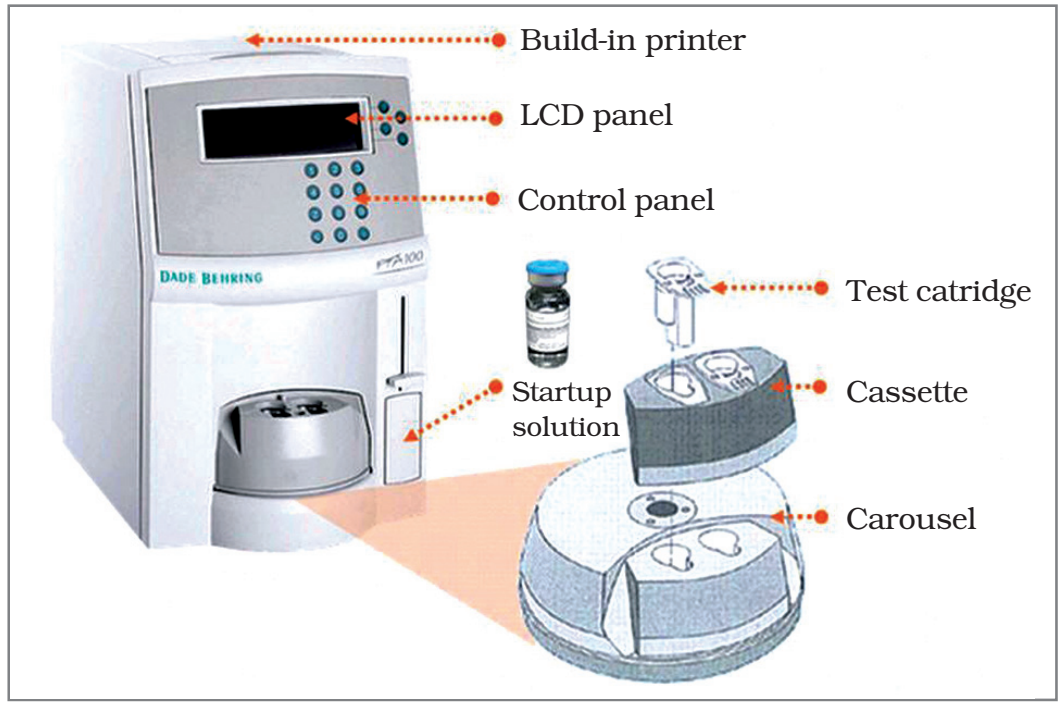

Fig.4 Schematic description of PFA-100 analyzer [16]

Blood is pipetted into the reservoir and aspirated through a capillary with a diameter of $200 \mathrm{~mm}$ with constant negative pressure resulting in high shear forces (5000-6000 s-1). The capillary ends in a membrane aperture with a diameter of $150 \mathrm{~mm}$. Platelets adhere at the aperture where they are activated by the collagen and then aggregate. The two agonists epinephrine and ADP enhance aggregation. Finally, a platelet plug occludes the aperture and blood flow stops. The time measured in sec. from the beginning of the test until formation of an occluding platelet plug is called closure time (CT) (Fig.5) [17]. If an occluding platelet plug does not form after $300 \mathrm{sec}$. the analysis is stopped.

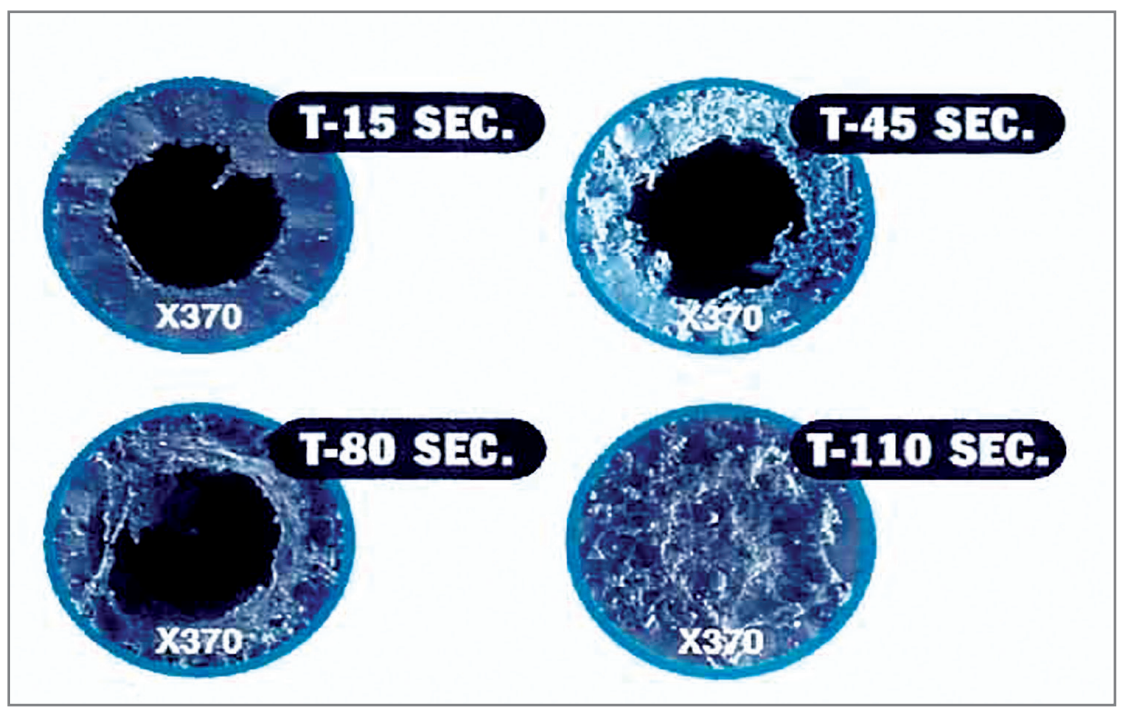

Fig.5 The micrographs showing the closing process in PFA 100 cartridge [17]

Citrated whole blood was aspirated from the sample container by means of a capillary opening, the platelets showing condition high shear stress. Membrane is coated with collagen, sub-endothelial protein, which is the starting template for the attachment of platelets (Fig.6) $[19,24]$. Platelet engraftment to collagen triggers an initial physiological stimulus for plate- 
let activation. Moreover, the membrane is covered by either epinephrine or ADP, which are other physiological antagonists (together with collagen) generally used for the induction of platelet aggregation.

Fig.6 Schematic diagram of PFA-100 instrument [19, 24]

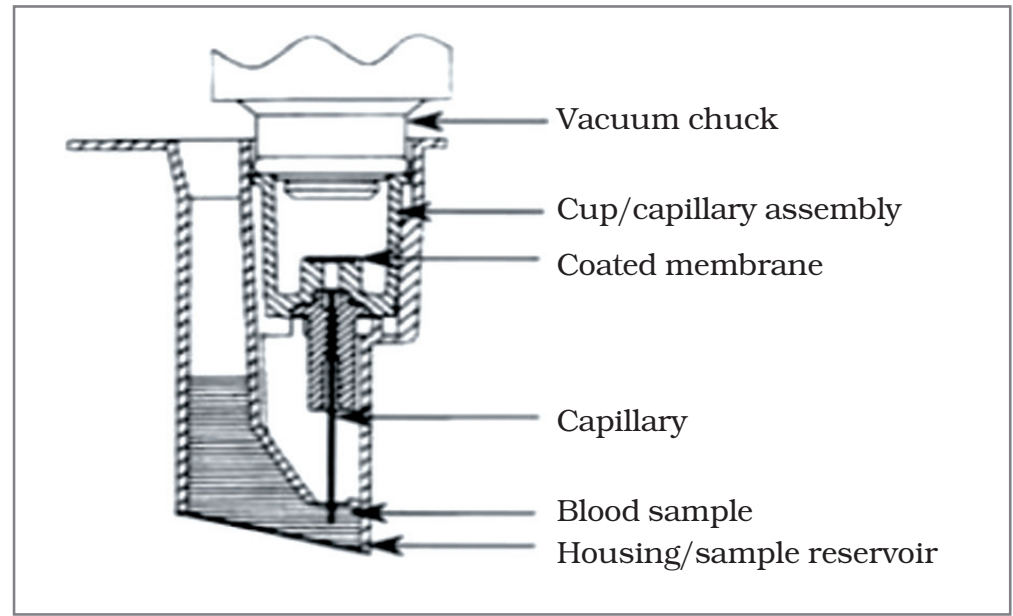

The PFA-100 system consists of a device and test cartridges, in which there is undergoing invitro simulation of the process of adhesion and platelet aggregation followed by vascular injury (Fig.7) [23].

Fig.7 The PFA-100 System simulates in Vivo Conditions [23]

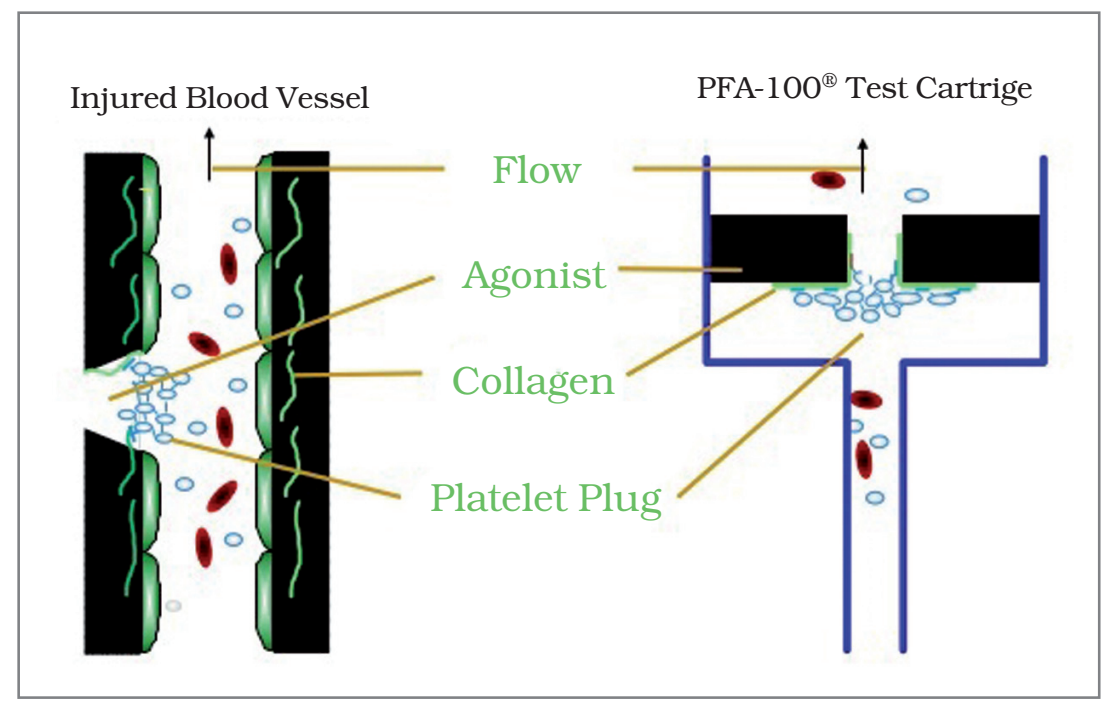

Platelet dysfunctions observed by PFA system are either acquired, congenital or induced by substances inhibiting platelets. The primary test cartridge Col / Epi is used to detect dysfunction induced by inner platelet disorder, vWCH, or by exposure to substances that inhibit platelets. Secondary test cartridge Col / ADP is used to determine whether the abnormal result measured by cartridge Col / Epi could be caused by effect of aspirin or by medicines containing aspirin. 


\section{RESULTS}

To determine PFA-100 reference intervals in the Slovak population, we evaluated CEPI and CADP CT in fifty, well-characterized healthy blood donors. We also studied the relationships between PFA-100 CT, gender and ABO blood group in these subjects. The results of graphic data analysis are depicted in Fig.8. - Fig. 16. The reference intervals for CEPI CT were 86 - $199 \mathrm{sec}$. The reference intervals for CADP CT were $42-119 \mathrm{sec}$. The reference intervals of CT in a group of women were as followed - CEPI cartridge: 86 - 171 sec., median: 138 , IQR : 55. 25. Reference intervals for CADP cartridge were: 58 - 102 sec., median : 90 , IQR : 12.75 ( Tab.1). The reference CT intervals in the group of men were as followed CEPI cartridge : 88 - 199 sec., median: 123.5, IQR: 42.25. The reference intervals for CADP CT were: 42 - 119 sec., median 87, IQR: 20 (Tab.1). Reference intervals for CEPI CT in blood group O were: 86 - 199 sec., median: 136, IQR: 27.5 ; the CADP CT : 42 - 119 sec., median: 91, IQR: 15.5 (Tab.2). The reference intervals for CEPI CT in blood group non O: 88 - 174 sec., median: 109, IQR: 43; CADP CT: 59 - 107 sec., median: 86, IQR: 19 (Tab.2). The results of CEPI CT between blood group $\mathrm{O}$ and blood group non-O were significantly different $(\mathrm{p}<0.05)$.

Table 1 Comparison of PFA-100 closure times between different variables (gender).

\begin{tabular}{|l|c|c|}
\hline Variables & \multicolumn{2}{|c|}{ Closure time in 3.2\% citrated blood (median, reference intervals, IQR) } \\
\hline Gender & CEPI & CADP \\
\hline Male $(\mathbf{n}=\mathbf{3 8})$ & $123.5(88-199)$ & $87(42-119)$ \\
& 42.25 & 20 \\
\hline Female $(\mathbf{n = 1 2})$ & $138(86-171)$ & $90(58-102)$ \\
& 55.25 & 12.75 \\
\hline p-values male vs. female & n.s. & n.s \\
\hline
\end{tabular}

Table 2 Comparison of PFA-100 closure times between different variables (blood group).

\begin{tabular}{|l|c|c|}
\hline Variables & \multicolumn{2}{|c|}{ Closure time in 3.2\% citrated blood (median, reference intervals, IQR) } \\
\hline ABO blood group & CEPI & CADP \\
\hline O $(\mathbf{n}=\mathbf{2 3})$ & $136(86-199)$ & $91(42-119)$ \\
& 27.5 & 15.5 \\
\hline Non O ( $\mathbf{n}=\mathbf{2 7})$ & $109(88-174)$ & $86(59-107)$ \\
& 43 & 19 \\
\hline p-values O vs. non O & $0.0265^{*}(\mathrm{p}<0.05)$ & n.s. \\
\hline
\end{tabular}




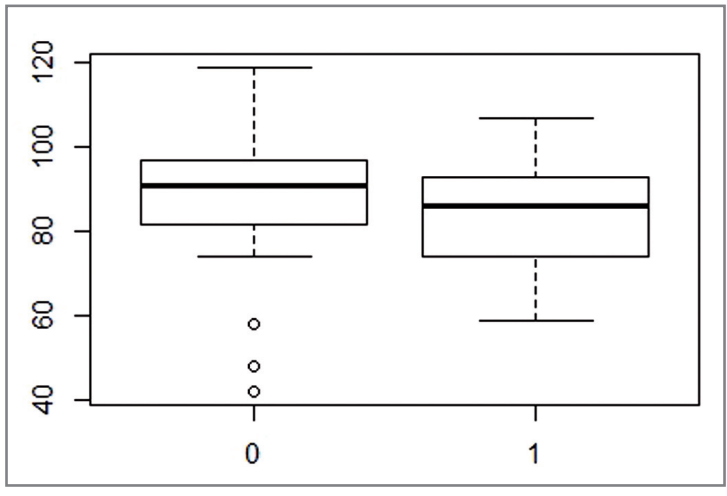

Fig.8 Comparision of PFA Col/EPI values between donors with blood group $\mathrm{O}$ and non $-\mathrm{O},(\mathrm{p}<0,05)$.



Fig.10 Analysis of distribution of PFA Col/EPI in blood donors with blood group $\mathrm{O}$.

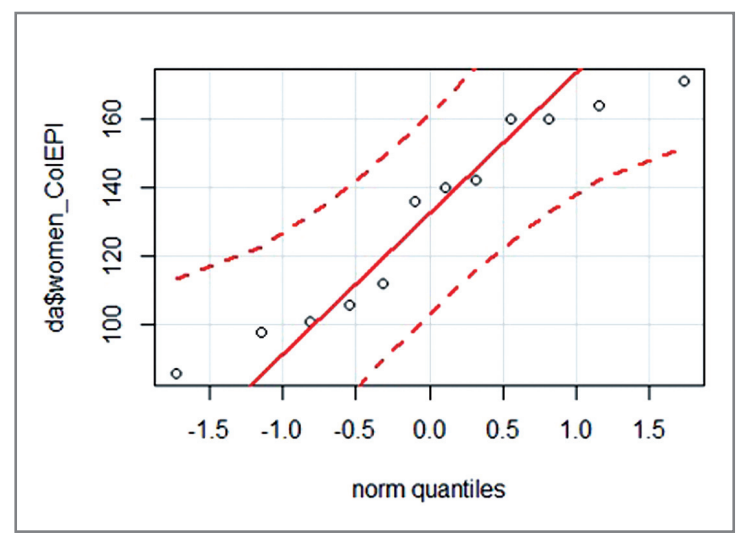

Fig.12 Analysis of distribution of PFA Col/EPI in blood donors with blood group non - O.



Fig.9 Comparision of PFA Col/ADP values between donors with blood group $\mathrm{O}$ and non $-\mathrm{O}$.

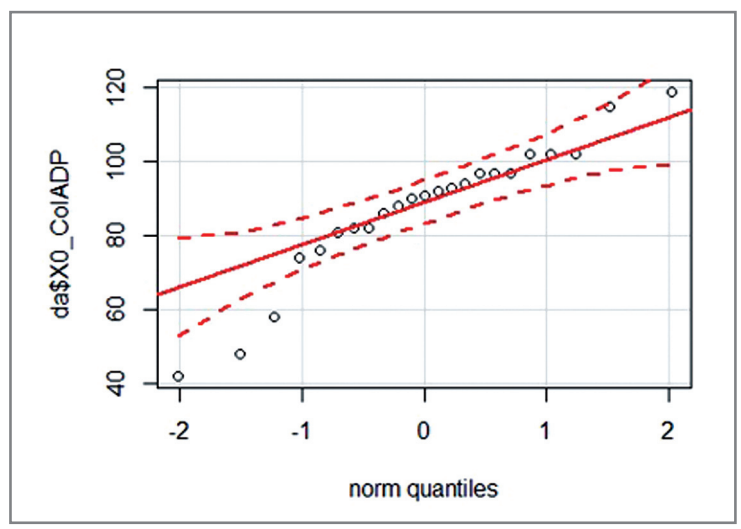

Fig.11 Analysis of distribution of PFA Col/ADP values in blood donors with blood group $\mathrm{O}$.

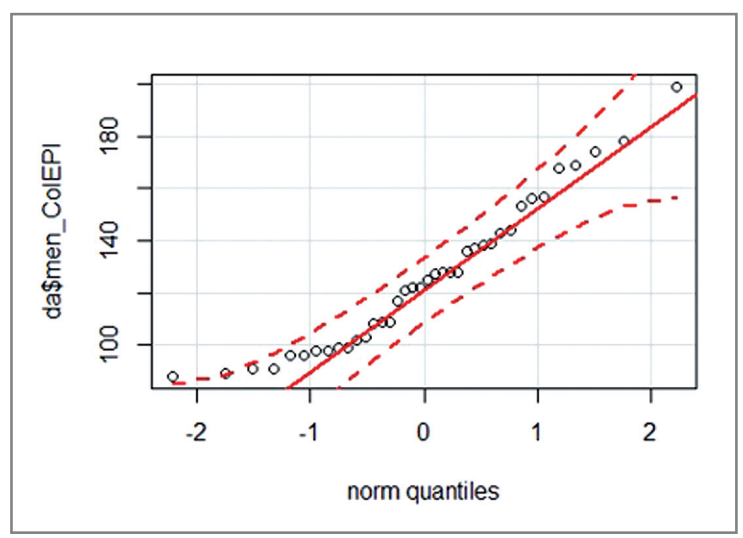

Fig.13 Analysis of distribution of PFA Col/ADP values in blood donors with blood group non - O. 




Fig.14 Analysis of distribution of PFA Col/EPI values in men.



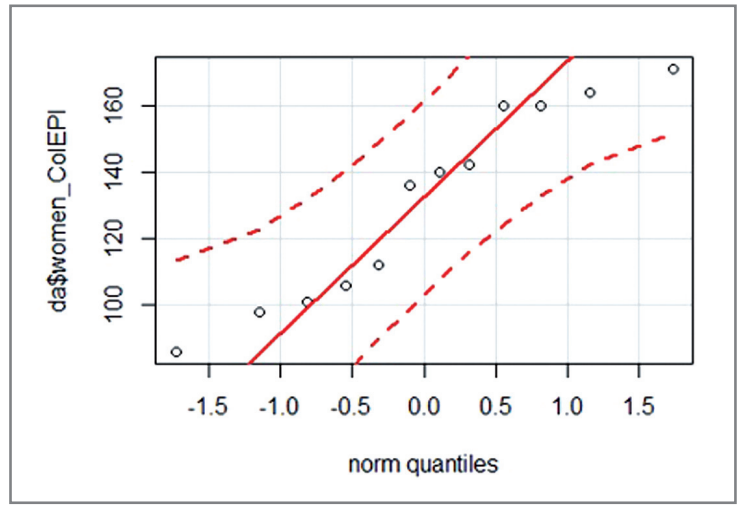

Fig. 15 Analysis of distribution of PFA Col/EPI values in women.

Fig. 16 Comparision of PFA Col/EPI values between men and women.

\section{DISCUSSION}

The appropriate approach for platelet function testing includes both the simple tests (e.g., PFA-100) and the detailed aggregation analysis, which are sensitive enough to capture the given defect. However, in platelet testing, the standardization has remained challenged by the relatively large blood volumes of blood (20-50 ml) needed, the sample freshness requirements for completion of the testing (within 3-4 hours), of light transmission aggregometry and whole blood aggregation and PFA-100, and difficulties in obtaining the same donor or donor pool for external quality assurance [7,20]. For PFA-100, the sensitivity for the correct diagnosis in case of normality ranges around $85 \%$, but the specificity for an abnormality around 55 to $75 \%$ with longstanding expertise leading to smaller variations [7,20]. There are comprehensive data to support the importance of validation and guidelines to be followed to assure the quality of the testing [20-22].

In accordance to these data, clinical performance of the PFA-100 device to screen for platelet dysfunction may be influenced by pre-analytical variables. Previous studies have found that age and gender have no effects on PFA-100 CT $[9,23]$ although men older than 55 year appeared to have shorter CT than younger men [23]. Diurnal variations in CT values have been reported, with samples collected in the morning having shorter CT than those collected the afternoon, particularly with the CEPI cartridge [5,24]. CT are longer for blood group O samples than 
for other blood groups [5-9], and are longer for blood samples collected in $3.8 \%$ vs $3.2 \%$ sodium citrate [4,8-10]. Although PFA-100 CT are prolonged when platelet count or hematocrit is significantly decreased [7-1 1], the cut-off value for abnormality of either has not been determined. PFA-100 CT are usually prolonged when platelet count is $<50.000 / \mu 1$ or hematocrit is $<25 \%$ [10]. In this context, the manufacturer notes that CT above the laboratory's established cut-off may reflect reduced platelet function caused by reduced hematocrit $(<35 \%)$ or platelet count $(<150.000 / \mu 1)$ influenced by gender and do not correlate with leukocyte count, platelet count, hematocrit, or platelet-associated parameters of routine CBCs [25].

As expected, our individuals with blood group O had longer PFA-100 CT than those with other blood groups, likely because vWF levels are lower in blood group $\mathrm{O}$ than in other blood groups $[26,27]$. However, for statistical evaluation using the paired Student's t-test and Wilcoxon rank sum test, we found significantly prolonged CT with only CEPI cartridge $(\mathrm{p}<0.05)$. Prolongation of CADP CT was not significant. PFA-100 CT is strongly dependent on the plasma vWF level [7-10,13]. During primary hemostasis, vWF serves as an adhesive molecule that binds the platelets to exposed collagen at sites of vascular injury. Thus, lower levels of vWF affect the formation of a hemostatic plug and typically prolong PFA-100 CT.

\section{CONCLUSION}

We measured and determined the reference intervals of CT for the PFA-100 in a group of healthy blood donors using CEPI and CADP cartridges. In our study there was confirmed the significant prolongation of CT in blood donors with blood group O, compared to the group of blood donors with blood group non O (CEPI : p < 0.05). The influence of gender, as another variable analyzed with CT, was not significant. Nevertheless, definitive conclusion, can't be ruled yet, due to the disproportionate representation of the gender (male group > female group) in the investigated blood donors population. In spite of this fact we conclude that the determination of reference intervals for CT PFA-100 in local laboratory has an irreplaceable role for further use of PFA-100 in everyday clinical practice. However, it should be interpreted with consideration of both the patient clinical presentation and laboratory variables such as blood groups.

\section{REFERENCES}

1. Primary hemostasis [cit 2017-01-29]. Available on webpage: https://www.google.sk/search?q= primaryhemostasis + pictures \&biw $=1366 \&$ bih $=622 \&$ noj $=1 \&$ tbm $=$ isch $\&$ tbo $=$ u\&source $=$ univ $\&$ sa $=X \& v e d=0 a$ hUKEwiSoZek 1fRAhVEtBoKHRRCA0kQ7AkINA\#imgrc=nmJjzJyX3BW8LM\%3A

2. Harrison P. Progress in the assessment of platelet function. Br J Haematol 2000; 111: 733-744.

3. Mammen EF, Comp PC, Gosselin R, Greenberg C, Hoots WK, Kessler CM, Larkin EC, Liles D, Nugent DJ. PFA-100 system: a new method for assessment of platelet dysfunction. Semin Thromb Hemost 1998; 24: 195-202.

4. Heilmann EJ, Kundu SK, Sio R, Garcia C, Gomez R, Christie DJ. Comparison of four commercial citrate blood collection systems for platelet function analysis by the PFA-100 system. Thromb Res 1997; 87: 159-164.

5. Dalby MC, Davidson SJ, Burman JF, Davies SW. Diurnal variation in platelet aggregation with the PFA100 platelet function analyzer. Platelets 2000; 11:320-324.

6. Lippi G, Franchini M, Brocco G, Manzato F. Influence of the ABO blood type on the platelet function analyzer PFA-100. Thromb Haemost 2001;85:369-370.

7. Lassila R. Platelet function tests in bleeding disorders. Semin Thromb Hemost 2016; 42(3): 185-190.

8. Jilma B. Platelet function analyzer (PFA-100): a tool to quantify congenital or acquired platelet dysfunction. J Lab Clin Med 2001; 138: 152-163.

9. Favaloro EJ. Clinical application of the PFA-100. Curr Opin Hematol 2002; 9: 407-415. 
10. Harrison P. The role of PFA-100 testing in the investigation and management of haemostatic defects in children and adults. Br J Haematol 2005; 130: 3-10.

11. Eugster M, Reinhart WH. The influence of the haematocrit on primary haemostasis in vitro. Thromb Haemost 2005;94:1213-1218.

12. Franchini M. The platelet-function analyzer (PFA-100) for evaluating primary hemostasis. Hematology 2005; 10: 177-181.

13. Kundu SK, Heilmann EJ, Sio R, et al. Description of an in vitro platelet function analyzer - PFA-100. Semin Thromb Hemost 1995; 21: 106-12.

14. Favaloro EJ, Bonar R. External quality assessment/proficiency testing and internal quality control for the PFA-100 and PFA-200: an update. Semin Thromb Hemost 2014; 40(2): 239-253.

15. Scavone M, Germanovich K, Fermia EA, et al. Usefulness of the INNOVANCE PFA P2Y test cartridge for the detection of patients with congenital defects of the platelet $\mathrm{P} 2 \mathrm{Y}_{12}$ receptor for adenosine diphosphate. Thromb Res 2014; 133(2): 254-256.

16. PFA-100 System [cit. 2017/01/29]. Available on webpage: https://usa.healthcare.siemens.com/hemostasis/systems/pfa-100

17. Platelet Research Laboratory. Techniques availables [cit. 2017/01/29]. Available on webpage: http://www.platelet-research.org/1/techniques_pfa.htm

18. Disorders of primary hemostasis: lecture 5 [cit. 2017/01/29]. Available on webpage: http:// www.slideshare.net/shababali 1/lecture-5-fall-2014-pdf

19. Kenichi A Tanaka The Assessment of platelet function. Journal Compilation @ 2006 LMS Transfusion Alternatives in Transfusion Medicine 2, 95- 105.Available on webpage: https://www.researchgate.net/publication/227625147 The_assessment of platelet function .

20. Favaloro EJ. Internal quality control and external quality assurance of platelet function tests. Semin Thromb Hemost 2009; 35(2): 139-149.

21. Zaffuto BJ, Coley GW, Connolly GC, et al. ABO immune complex formation and impact on platelet function, red cell structural integrity and haemostasis: an in vitro model of ABO non-identical transfusion. Vox Sang 2016; 110(3): 219-226.

22. Peerschke EI, Castellone DD, Stroobants AK, et al. Reference range determination for whole- blood platelet aggregation using Multiplate analyzer. Am J Clin Pathol 2014; 142(5): 647-656.

23. Poujoul C,Nurden A,Papponeau A, Heilmann E, Nurden P. Ultrastructural analysis of the distribution of von Willebrand factor and fibrinogen in platelet aggregates formed in the PFA-100. Platelets 1998; 9: 381-9.

24. Young-Uk Cho, Seongsoo Jang, Chan-Jeoung Park, . Variables that affect platelet function analyzer100 (PFA-100) closure times and establishment of reference intervals in Korean adults. Ann Clin Lab Sci 2008 ;38(3):247-53.

25. Harrison P, Robinson MS, Mackie IJ, Joseph J, McDonald SJ, Liesner R, Savidge GF, Pasi J, Machin SJ. Performance of the platelet function analyzer PFA-100 in testing abnormalities of primary haemostasis. Blood Coagul Fibrinolysis 1999; 10: 25-31.

26. DiPaola J, Federici AB, Mannucci PM, Canciani MT, Kritzik M, Kunicki TJ, Nugent D. Low platelet alpha2beta1 levels in type I von Willebrand disease correlate with impaired platelet function in a high shear stress system. Blood 1999; 93: 3578-82.

27. Marshall PW, Williams AJ, Dixon RM, Growcott JW, Warburton S, Armstrong J, Moores J. A comparison of the effects of aspirin on bleeding time measured using the Simplate method and closure time measured using the PFA-100, in healthy volunteers. Br J Clin Pharmacol 1997; 44: 151-5.

\section{Acknowledgement}

This study was supported by CEPV II (ITMS 26220120036), APVV 0-222-11 and Vega 1/0168/16 projects.

Received: February, 2, 2017

Accepted: March, 16, 2017 